\title{
Methylphenidate Hydrochloride
}

National Cancer Institute

\section{Source}

National Cancer Institute. Methylphenidate Hydrochloride. NCI Thesaurus. Code C646.

The hydrochloride salt of the synthetic central nervous system stimulant

methylphenidate. Methylphenidate appears to activate the brain stem arousal system and cortex to produce its stimulant effect and, in some clinical settings, may improve cog nitive function. 